\title{
Modificação térmica nas propriedades físicas da madeira
}

\author{
Thermal modification on the physical properties of wood
}

\author{
Walmir Marques de Menezes $^{{ }^{*}}$ Elio José SantiniI ${ }^{\mathrm{II}}$ Joel Telles de Souza \\ Darci Alberto Gatto ${ }^{\mathrm{III}}$ Clovis Roberto Haselein ${ }^{\mathrm{II}}$
}

\section{RESUMO}

O objetivo desta pesquisa foi avaliar o efeito da modificação térmica nas propriedades físicas da madeira de Corymbia citriodora e Eucalyptus saligna, comparando com a testemunha (madeira não tratada). Para isso, foram amostradas 3 árvores de cada espécie, com aproximadamente 40 anos de idade, das quais foram retiradas as duas primeiras toras de 3 metros de comprimento. A modificação térmica da madeira foi conduzida por meio do emprego de temperaturas finais de $140^{\circ} \mathrm{C}, 160^{\circ} \mathrm{C} e$ $180^{\circ} \mathrm{C}$ durante 2,5 horas, mais a testemunha (sem tratamento), utilizando estufa com circulação de ar forçada. Os ensaios das propriedades físicas foram realizados segundo os procedimentos estabelecidos na norma ASTM e adaptações (ASTM 143-94, 2000), e consistiram na avaliação da umidade de equilíbrio, perda de massa, coeficiente de inchamento total (linear e o volumétrico) e coeficiente de anisotropia. No geral, os resultados evidenciaram que a modificação térmica foi eficiente na redução da umidade de equilíbrio, na perda de massa, na diminuição do inchamento total e do coeficiente de anisotropia de ambas as espécies, sendo os valores mais expressivos observados nos tratamentos de maiores temperaturas.

Palavras-chave: umidade de equilíbrio, perda de massa, inchamento total, estabilidade dimensional.

\section{ABSTRACT}

The objective of this research was to evaluate the effect of thermal change in the physical properties of wood Corymbia citriodora and Eucalyptus saligna, compared with the control (untreated wood). For that were sampled three trees of each species, with approximately 40 years of age, which were taken the first two logs 3 feet long. The thermal modification of wood was conducted by employing final temperature of $140^{\circ} \mathrm{C}, 160^{\circ} \mathrm{C}$ and $180^{\circ} \mathrm{C}$ for 2.5 hours, over the control (no treatment) using an oven with forced air circulation. Tests of physical properties were performed according to established procedures in ASTM and adaptations (ASTM 143-94, 2000), and consisted in evaluating the equilibrium moisture content, weight loss, swelling coefficient of total (linear and volumetric) and coefficient anisotropy. Overall, the results showed that the thermal modification was effective in reducing the moisture balance, weight loss, decrease in swelling and total anisotropy coefficient of both species, with the higher values observed in the treatments of higher temperatures.

Key words: modification thermal, weight loss, swelling total, increased dimensional stability.

\section{INTRODUÇÃO}

A madeira é um material higroscópico, devido à estrutura química da parede celular, onde contém diversos polímeros de celulose, hemicelulose e lignina, que formam os grupos hidroxílicos. Dentre esses polímeros, a hemicelulose é a mais hidrófila, favorecendo a troca de água com o meio, que contribui para a variação dimensional da madeira (HILL, 2006).

A água na madeira é classificada em duas formas: água livre ou de capilaridade e água higroscópica ou de adesão. Ela está presente na madeira na forma líquida ou vapor. A água livre localizada nos vasos, canais e lúmen das células está sujeita a forças capilares, e teoricamente é fácil de ser retirada. Ao contrário da anterior, a água higroscópica

\footnotetext{
IPrograma de Pós-graduação em Engenharia Florestal (PPGEF), Centro de Ciências Rurais (CCR), Universidade Federal de Santa Maria (UFSM), 97105-900, Santa Maria, RS, Brasil. E-mail: walmirmenezessm@hotmail.com. *Autor para correspondência.

IIDepartamento de Ciências Florestais, CCR, UFSM, Santa Maria, RS, Brasil.

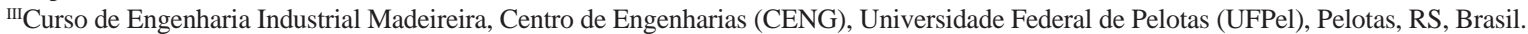
Recebido 21.01.13 Aprovado 07.11.13 Devolvido pelo autor 27.03.14 CR-2013-0082.R1
} 
é mais difícil de ser removida da madeira, pois esse tipo de água está aderida por ligações de ponte de hidrogênio aos grupos hidroxílicos nas cadeias da celulose, hemicelulose e, em menor extensão, na lignina (SKAAR, 1972; SIAU, 1995).

Logo, em uma secagem da madeira, a água livre contida no lúmen das células é a primeira a ser removida. Após toda essa água livre ser removida da madeira, a parede celular ainda estará saturada de água, então, esse momento é conhecido como ponto de saturação das fibras (PSF) (HAYGREEN e BOWYER, 1996). Esse ponto varia de espécie para espécie, com valores geralmente entre 28 e $30 \%$. Abaixo desse ponto, é o momento crítico para a secagem da madeira, que causa maiores defeitos irreversíveis nela.

Devido às limitações da madeira, há muitos anos, os cientistas pesquisam novas técnicas para melhorar as propriedades da madeira e assim aumentar a sua utilização e durabilidade natural. Dessa forma, diferentes estudos vêm sendo empregados e, dentre eles, surgiu o tratamento térmico. Este método é um dos mais antigos, mais fáceis e mais baratos para melhorar as propriedades físicas da madeira como a higroscopicidade e a estabilidade dimensional (ARAÚJO, 2010).

A redução da instabilidade dimensional da madeira após a modificação térmica é explicada por diversos autores STAMM (1964), CALONEGO (2009), como sendo um fenômeno químico que ocasiona a degradação de alguns constituintes químicos fundamentais da estrutura anatômica da madeira.

A modificação térmica basicamente é um processo pelo qual a madeira é submetida a altas temperaturas, inferiores a sua combustão, que provocam o início da degradação de seus constituintes químicos fundamentais, sobretudo as hemiceluloses que são, dentre eles, os mais sensíveis à ação do calor (BRITO et al., 2006).

As temperaturas utilizadas no processo estariam entre 100 e $250^{\circ} \mathrm{C}$, segundo os autores VOVELLE \& MELLOTTEE (1982), o que resulta na obtenção de um produto sólido, apresentando características diferenciadas, comparativamente à sua madeira original, algumas delas podendo tornar-se interessantes para aplicações diversas da madeira.

A modificação térmica é empregada em escala industrial na Europa, inclusive com patentes em alguns países, como a Finlândia, França, Holanda e Alemanha. As principais diferenças entre esses tratamentos são: teor de umidade inicial da madeira, as temperaturas e tempo em que as peças ficam expostas ao tratamento, utilização de oxigênio ou nitrogênio, presença ou ausência de vapor, processo seco ou úmido e a utilização de óleos.

Dessa forma, o presente estudo teve como objetivo avaliar o efeito da modificação térmica nas propriedades físicas da madeira de Corymbia citriodora e Eucalyptus saligna, por meio dos ensaios de umidade de equilíbrio, redução de massa, coeficiente de inchamento total (linear e o volumétrico) e coeficiente de anisotropia. Além de melhorar as propriedades da madeira, a aplicação da técnica busca aumentar sua qualidade, agregar valor aos produtos e ampliar suas possibilidades de utilização.

\section{MATERIAL E MÉTODOS}

As espécies utilizadas neste estudo foram Corymbia citriodora e Eucalyptus saligna, coletadas em plantios pertencentes à Fundação Estadual de Pesquisa Agropecuária - FEPAGRO FLORESTAS, localizada no município de Santa Maria - RS. De cada espécie, foram utilizadas três árvores com aproximadamente 40 anos de idade, das quais seccionaram-se as duas primeiras toras com 3,0m de comprimento, para a confecção das tábuas, com dimensões aproximadas de 2,5x15,0x280cm de espessura, largura e comprimento, respectivamente, pelo método de desdobro tangencial. Em seguida, as tábuas foram transportadas para o Laboratório de Produtos Florestais (LPF), da Universidade Federal de Santa Maria, onde o material foi preparado para a condução do estudo.

Antes do tratamento de modificação térmica, as tábuas foram submetidas à secagem prévia até o teor de umidade de $10 \%$ em uma estufa piloto, com capacidade nominal de aproximadamente $1 \mathrm{~m}^{3}$ de madeira serrada, utilizando-se um programa de secagem com temperatura inicial de $39^{\circ} \mathrm{C}$ e $40^{\circ} \mathrm{C}$, temperatura final $68^{\circ} \mathrm{C}$ e $67^{\circ} \mathrm{C}$ e potencial de secagem (relação umidade da madeira/umidade de equilíbrio) de 2,1 para as madeiras de $\boldsymbol{C}$. citriodora e $\boldsymbol{E}$. saligna, respectivamente.

Para a execução do tratamento de modificação térmica, utilizou-se uma estufa elétrica, de convecção forçada e controle digital de temperatura. Para a condução do tratamento, corpos-de-prova com dimensões de 2,0x 2,0x32cm de espessura, largura e comprimento, respectivamente, foram submetidos a temperaturas finais de $140^{\circ} \mathrm{C}, 160^{\circ} \mathrm{C}$ e $180^{\circ} \mathrm{C}$ durante 2,5 horas, conforme ilustrado na figura 1 . Previamente ao tratamento, usando metodologia proposta por CALONEGO (2009), a madeira foi submetida à temperatura inicial de $100^{\circ} \mathrm{C}$ por 14 horas, com o 


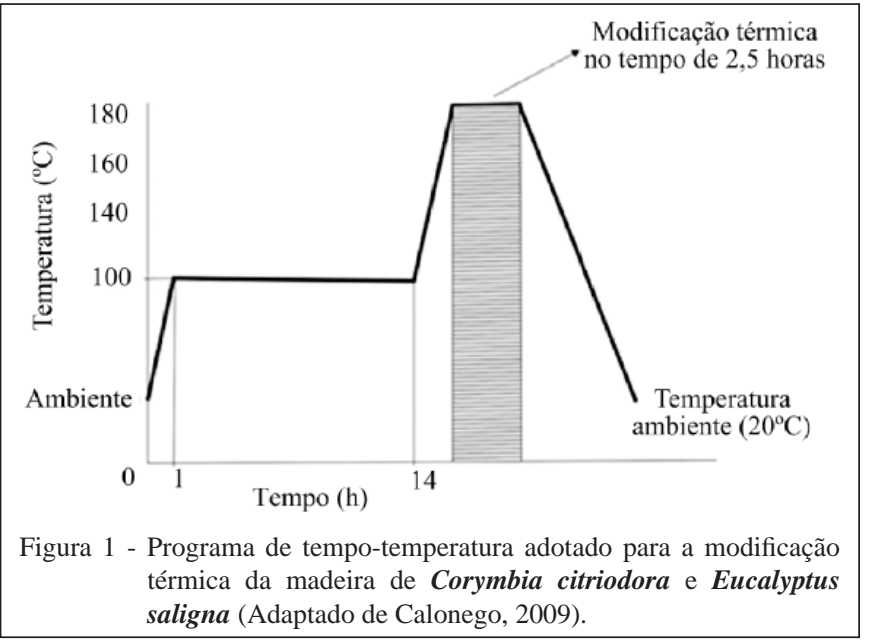

objetivo de reduzir o teor de umidade para próximo de $3 \%$, visando a evitar provável problema de expansão de vapor de água e rompimento da parede celular. Logo após ao tratamento, os corpos-de-prova foram redimensionados para 2,0x2,0x8,0cm com orientação na direção radial, tangencial e axial, respectivamente, para a realização dos ensaios físicos, os quais foram conduzidos de acordo com a norma ASTM 14394 e adaptações (ASTM, 2000). Posteriormente, foram armazenados na câmara climática $\left(20^{\circ} \mathrm{C}\right.$ de temperatura e 65\% umidade relativa), para a estabilização do seu teor de umidade.

Ao término do tratamento de modificação térmica, a estufa foi desligada e os corpos-deprova foram mantidos até o resfriamento natural de aproximadamente $20^{\circ} \mathrm{C}$. Antes e após a aplicação da modificação térmica, as peças foram pesadas com balança digital de precisão centesimal e medidas com auxílio de um paquímetro com precisão decimal, para a obtenção da massa e volume, respectivamente, usados posteriormente na determinação das seguintes propriedades: umidade de equilíbrio, redução de massa, coeficiente de inchamento total e coeficiente de anisotropia de inchamento.

No experimento foram utilizados oito tratamentos, sendo as temperaturas finais de $140^{\circ} \mathrm{C}$, $160^{\circ} \mathrm{C}, 180^{\circ} \mathrm{C}$ e mais a testemunha, para as espécies Eucalyptus saligna e Corymbia citriodora, com um total de 15 corpos-de-prova por tratamento, num total de 60 corpos-de-prova para cada espécie estudada.

Os dados das propriedades físicas foram organizados no programa Excel $2007^{\circledR}$, analisados e processados estatisticamente pelo software Statgraphics Centurion XV.II. No caso de serem detectadas diferenças significativas, as médias foram comparadas pelo teste de Tukey em 5\% de probabilidade de erro. Os efeitos dos tratamentos foram avaliados com auxílio de análise de variância.

\section{RESULTADOS E DISCUSSÃO}

Na tabela 1, são apresentados os valores médios da umidade de equilíbrio resultante dos ensaios do tratamento térmico mais as testemunhas, da madeira de Eucalyptus saligna e Corymbia citriodora. Observa-se estatisticamente que as médias da umidade de equilíbrio são diferentes entre os tratamentos. Logo, houve interação significativa entre o método e a temperatura de tratamento térmico.

A madeira não tratada termicamente apresentou umidade de equilíbrio maior para as duas espécies, obtendo-se um valor de $12,7 \%$ para $\boldsymbol{E}$. saligna e $12,1 \%$ C. citriodora, quando comparada com as madeiras submetidas ao tratamento térmico, comprovando o efeito do tratamento térmico na higroscopicidade da madeira. As maiores reduções da umidade de equilíbrio em comparação com a madeira testemunha foram encontradas nos tratamentos de maiores temperaturas $\left(180^{\circ} \mathrm{C}\right)$, com reduções de 44,6\% e 54,3\%, respectivamente, para $\boldsymbol{E}$. saligna e $\boldsymbol{C}$. citriodora. Esses valores são semelhantes ao exposto por JÄMSÄ e VIITANIEMI (2001), que, ao submeter a madeira ao tratamento térmico, encontraram reduções de até $50 \%$ do teor de umidade higroscópico, atribuindo essa diminuição à perda de sítios de adsorção e à degradação parcial das hemiceluloses (grupos hidroxílicos). Segundo BOONSTRA e TJEERDSMA (2006), a menor disponibilidade de grupos hidroxílicos é ocasionada pela cristalização da celulose e aumento das ligações cruzadas na lignina, que, consequentemente, aumenta a estabilidade dimensional. 
Tabela 1 - Umidade de equilíbrio média em câmara climática e redução de massa das madeiras de Eucalyptus saligna e Corymbia citriodora tratadas termicamente.

\begin{tabular}{|c|c|c|c|c|c|c|}
\hline \multirow{2}{*}{ Espécie } & \multirow{2}{*}{ Tratamentos } & \multicolumn{5}{|c|}{---------------------Umidade de equilíbrio a 12\%---------------------- ---'Redução de massa----. } \\
\hline & & $\mathrm{CV}(\%)$ & Média (\%) & Redução em relação à testemunha (\%) & C.V. (\%) & Média (\%) \\
\hline \multirow{4}{*}{ Eucalytus saligna } & Testemunha & 4,66 & $12,7 \mathrm{a}$ & - & - & - \\
\hline & $140^{\circ} \mathrm{C}$ & 10,18 & $8,8 b$ & 30,3 & 3,61 & $8,83 a$ \\
\hline & $160^{\circ} \mathrm{C}$ & 17,77 & 7,9c & 37,8 & 3,62 & $9,79 b$ \\
\hline & $180^{\circ} \mathrm{C}$ & 6,70 & $7,0 \mathrm{~d}$ & 44,6 & 4,75 & $10,93 c$ \\
\hline \multirow{4}{*}{ Corymbia citriodora } & Testemunha & 2,52 & $12,1 \mathrm{a}$ & - & & \\
\hline & $140^{\circ} \mathrm{C}$ & 5,93 & $7,9 \mathrm{~b}$ & 34,4 & 5,48 & $6,30 a$ \\
\hline & $160^{\circ} \mathrm{C}$ & 5,92 & $7,1 \mathrm{c}$ & 41 & 9,48 & $8,14 b$ \\
\hline & $180^{\circ} \mathrm{C}$ & 5,17 & $5,5 d$ & 54,3 & 6,29 & $12,12 \mathrm{c}$ \\
\hline
\end{tabular}

CV = coeficiente de variação, \%; médias seguidas pela mesma letra, para a mesma espécie, não diferem estaticamente em nível de 5\% de significância pelo teste de Tukey.

De outro lado, a menor redução foi observada para as menores temperaturas $\left(140^{\circ} \mathrm{C}\right)$, quando se obtiveram valores de umidade de equilíbrio 30,3\% e 34,4\% menores que a testemunha, para as duas espécies. A diminuição da umidade de equilíbrio e a perda de massa resultam no aumento da estabilidade dimensional da madeira, que é uma característica positiva para sua utilização. A tabela 1 mostra que as maiores reduções de massa foram 10,9 e 12,1\% para as madeiras de $\boldsymbol{E}$. saligna e $\boldsymbol{C}$. citriodora, respectivamente, obtidos no tratamento de $180^{\circ} \mathrm{C}$. Esses valores estão próximos aos obtidos por MODES (2010), que encontrou perda de massa entre 11,7\% e 12,9\% para Eucalyptus grandis e Pinus taeda, respectivamente. Entretanto, são superiores aos obtidos por BRITO et al. (2006) para as madeiras de $\boldsymbol{P}$. caribaea var. hondurensis e $\boldsymbol{E}$. saligna, tratadas a $180^{\circ} \mathrm{C}$ em condições secas. De outro lado, as menores perdas de massa encontradas neste estudo para as madeiras de $\boldsymbol{E}$. saligna e $\boldsymbol{C}$. citriodora foram $8,8 \%$ e $6,3 \%$, respectivamente, para as menores temperaturas. Todas as médias de perda de massa foram significativas estatisticamente entre si.

Segundo alguns autores (BRITO et al., 2006; MODES, 2010), a redução de massa da madeira tratada termicamente ocorre, possivelmente, pela degradação de alguns constituintes fundamentais da parede celular, em especial a hemicelulose, que é a mais sensível ao calor.

A tabela 2 apresenta os valores médios do inchamento total (linear e volumétrico) e o coeficiente de anisotropia das madeiras de Eucalyptus saligna e Corymbia citriodora em relação aos tratamentos. No geral, os valores do inchamento total e o coeficiente de anisotropia modificado termicamente foram estatisticamente diferentes. Apenas os valores de inchamento radial e longitudinal na temperatura de $140^{\circ} \mathrm{C}$, para ambas as espécies, e longitudinal na temperatura de $160^{\circ} \mathrm{C}$ para a espécie de Eucalyptus saligna, não apresentaram a mesma tendência.

Valores diferentes ao exposto na tabela 2, para ambas as espécies, foram encontrados por BATISTA (2012), que obteve para as testemunhas, médias de $8,88 \%, 4,18 \%$ e $0,34 \%$, e para a temperatura de $180^{\circ} \mathrm{C}$ valores de $5,45 \%, 3,05 \%$ e $0,27 \%$, para as orientações tangencial, radial e longitudinal, respectivamente. Entretanto, esses resultados se assemelham aos obtidos por OLIVEIRA \& SILVA (2003) e CHAUHAN \& AGGARWAL (2004), que encontraram valores de inchamento linear tangencial, radial e longitudinal variando entre 3,5 e 15,0\%, entre 2,4 e $11,0 \%$ e entre 0,1 e $1,0 \%$, respectivamente.

Os valores médios obtidos neste estudo, para o tratamento testemunha de inchamento volumétrico foram de 20,63\% e 21,23\%, das espécies de Eucalyptus saligna e Corymbia citriodora, respectivamente. As maiores reduções médias do inchamento volumétrico, ou seja, as menores variações na madeira ocorreram para ambas às espécies, no tratamento de $180^{\circ} \mathrm{C}$, com valores próximos de 32,96\% para Eucalyptus saligna e 32,27\% para Corymbia citriodora.

Os resultados de reduções do inchamento volumétrico obtidos neste estudo de 12,19\%, 21,44\%, $32,96 \%$ de Eucalyptus saligna e 11,45\%, 19,82\%, $32,27 \%$ para Corymbia citriodora, respectivamente, para as temperaturas de $140^{\circ} \mathrm{C}, 160^{\circ} \mathrm{C}$ e $180^{\circ} \mathrm{C}$, estão semelhantes aos alcançados por CALONEGO (2009), que obteve reduções de $14,5 \%, 23,2 \%$ e $29,6 \%$, respectivamente, para as temperaturas de $140^{\circ} \mathrm{C}$, $160^{\circ} \mathrm{C}$ e $180^{\circ} \mathrm{C}$, para a espécie de Eucalyptus grandis. 
Tabela 2 - Inchamento total e coeficiente de anisotropia das madeiras de Eucalyptus saligna e Corymbia citriodora tratadas termicamente.

\begin{tabular}{|c|c|c|c|c|c|c|c|}
\hline \multirow[b]{2}{*}{ Espécie } & \multirow[b]{2}{*}{ Tratamentos } & \multicolumn{5}{|c|}{--------------------------------------Inchamento total (\%)------------------------------------- } & \multirow{2}{*}{$\begin{array}{l}\text { Coeficiente de } \\
\text { anisotropia }\end{array}$} \\
\hline & & Radial & Tangencial & Longitudinal & Volumétrico & $\begin{array}{l}\text { Redução } \\
\text { volumétrica }\end{array}$ & \\
\hline \multirow{4}{*}{ Eucalytus saligna } & Testemunha & 7,33 а $(10,58 \%)$ & 12,02 a $(9,05 \%)$ & 0,32 а $(7,59 \%)$ & 20,63 а $(8,76 \%)$ & - & $1,65 a(11,19 \%)$ \\
\hline & $140^{\circ} \mathrm{C}$ & $7,03 \mathrm{ab}(7,85)$ & $10,0 \mathrm{~b}(5,75 \%)$ & $0,32 \mathrm{ab}(5,85 \%)$ & $18,11 \mathrm{~b}(4,78 \%)$ & 12,19 & $1,43 \mathrm{~b}(9,44 \%)$ \\
\hline & $160^{\circ} \mathrm{C}$ & 6,70 b $(9,67 \%)$ & 8,55 с $(6,33 \%)$ & 0,32 a $(9,03 \%)$ & 16,20 с $(7,04 \%)$ & 21,44 & 1,28 с $(8,13 \%)$ \\
\hline & $180^{\circ} \mathrm{C}$ & 6,0 с $(18,08 \%)$ & $7,06 \mathrm{~d}(13,40 \%)$ & $0,30 \mathrm{~b}(6,05 \%)$ & 13,83 d (10,87\%) & 32,96 & 1,22 с $(23,13 \%)$ \\
\hline \multirow{4}{*}{ Corymbia citriodora } & Testemunha & 7,74 a $(8,0 \%)$ & 12,13 a $(5,52 \%)$ & 0,35 a $(6,52 \%)$ & 21,23 a $(5,49 \%)$ & - & 1,57 a $(8,05 \%)$ \\
\hline & $140^{\circ} \mathrm{C}$ & $7,57 \mathrm{ab}(3,96 \%)$ & 10,07 b (5,81\%) & 0,34 a $(5,27 \%)$ & 18,80 b $(4,24 \%)$ & 11,45 & 1,33 b $(6,28 \%)$ \\
\hline & $160^{\circ} \mathrm{C}$ & 7,03 b (16,08\%) & 8,98 с $(13,58 \%)$ & 0,32 b $(9,45 \%)$ & 17,02 с $(14,43 \%)$ & 19,82 & 1,28 bc $(7,48 \%)$ \\
\hline & $180^{\circ} \mathrm{C}$ & 6,07 с $(13,07 \%)$ & 7,52 d (13,41\%) & 0,29 с $(8,20 \%)$ & 14,38 d $(13,26 \%)$ & 32,27 & $1,24 \mathrm{c}(3,90 \%)$ \\
\hline
\end{tabular}

Números entre parênteses correspondem ao coeficiente de variação, \%; médias seguidas pela mesma letra, para a mesma espécie, não diferem estaticamente ao nível de 5\% de significância pelo teste de Tukey.

Os valores médios de coeficiente de anisotropia da tabela 2 demonstram que a modificação térmica diminui significativamente o fator anisotrópico (relação T/R) das madeiras de Eucalyptus saligna e Corymbia citriodora. A testemunha diferiu estatisticamente ao nível de 5\% de probabilidade dos demais tratamentos. Os maiores valores médios foram obtidos pelas testemunhas, com 1,65 para Eucalyptus saligna e 1,57 para Corymbia citriodora. Os menores valores médios para ambas as espécies foram obtidos na temperatura de $180^{\circ} \mathrm{C}$. Valores próximos foram alcançados por CALONEGO (2009), com 1,70, 1,56, 1,61 e 1,54 para a testemunha e as temperaturas $140^{\circ} \mathrm{C}, 160^{\circ} \mathrm{C}$ e $180^{\circ} \mathrm{C}$, respectivamente.

Segundo STAMM (1964) e CALONEGO (2009), alguns constituintes químicos fundamentais da estrutura anatômica da madeira, como, por exemplo, a celulose, sofre cristalização, devido à destruição dos grupos hidroxílicos livres na região amorfa desta, e a reticulação dos polímeros que compõem a madeira durante o seu tratamento. As hemiceluloses também são degradadas e, com isso, tornam a madeira menos higroscópica, resultando consequentemente na redução do inchamento total e coeficiente de anisotropia da madeira.

\section{CONCLUSÃO}

O tratamento de modificação térmica aplicado nas madeiras de Corymbia citriodora e Eucalyptus saligna evidenciou uma redução significativa na umidade de equilíbrio, em comparação com a testemunha, acompanhada de um aumento da perda de massa, conforme a temperatura foi aumentada entre 140 e $180^{\circ} \mathrm{C}$. Da mesma forma, a capacidade de inchamento total da madeira e o coeficiente de anisotropia tiveram suas maiores reduções nas maiores temperaturas de tratamento. Esses resultados evidenciam o efeito marcante do tratamento térmico nas propriedades físicas e, consequentemente, na estabilidade dimensional da madeira.

\section{REFERÊNCIAS}

ASTM (AMERICAN SOCIETY FOR TESTING AND MATERIALS). ASTM D 143-94: standard methods of testing small clear specimens timber. Philadelphia, 2000. 31p.

ARAUJO, S. de O. Propriedades de madeiras termorretificadas. 2010. 77f. Tese (Doutorado em Ciência Florestal) - Departamento de Engenharia Florestal, Universidade Federal de Viçosa, Viçosa, MG.

BATISTA D.C. Modificação térmica da madeira de Eucalyptus grandis em escala industrial pelo processo brasileiro Vap HolzSysteme $^{\circledR}$. 2012. 339f. Tese (Doutorado em Engenharia Florestal) - Programa de Pós-graduação em Engenharia Florestal, Universidade Federal do Paraná, Curitiba, PR.

BOONSTRA, M.J.; TJEERDSMA, B.F. Chemical analysis of heat-treated softwoods. Holz Roh-Werkst, v.64, n.1, p.203-212, 2006. Disponível em: <http://link.springer.com/ article/10.1007\%2Fs00107-005-0078-4\#page-1>. Acesso 10 novembro de 2012. doi: 10.1007/s00107-005-0078-4.

BRITO, J.O. et al. Densidade básica e retratibilidade da madeira de Eucalyptus grandis submetida a diferentes temperaturas de termorretificação. Cerne, Lavras, v.12, n.2, p.181-188, 2006. Disponível em: <http://www.dcf.ufla.br/cerne/artigos/10-02 20094432v12_n2_artigo\%2009.pdf> Acesso 25 setembro de 2012.

CALONEGO, F.W. Efeito da termorretificação nas propiedades físicas, mecânicas e na resistência a fungos deterioradores da madeira de Eucalyptus grandis Hill ex. Maiden. 2009. 149f. Tese (Doutorado em Agronomia) - Faculdade de Ciências Agronômicas, Universidade Estadual de São Paulo, Botucatu, SP.

CHAUHAN, S.S.; AGGARWAL, P. Effect of moisture sorption state on transverse dimensional changes in wood. Holz als Roh- 
und Werkstoff, Berlin, v.62, n.1, p.51-56, 2004. Disponível em: <http://link.springer.com/article/10.1007\%2Fs00107-003-0437y\#page-1> Acesso 27 setembro de 2012. doi: 10.1007/s00107003-0437-y.

HAYGREEN, J.G.; BOWYER, J.L. Forest products and wood science: an introduction. Ames: Iowa State University, 1996. 484p.

HILL, C. Wood modification: chemical, thermal and other processes. West Sussex: John Wiley \& Sons, 2006. 233p.

JÄMSÄ, S.; VIITANIEMI, P. Heat treatment of wood: better durability with out chemicals. In: RAPP, A.O. Review on heat treatments of wood. In: SPECIAL SEMINAR: ENVIRONMENTAL OPTIMISATION OF WOOD PROTECTION, 2001. Antibes, France. Proceedings... Antibes, France: COST ACTION E 22, 2001. p.20-25.

MODES. K.S. Efeito da retificação térmica nas propriedades físico-mecânicas e biológicas das madeiras de Pinus taeda e Eucalyptus grandis. 2010. 99f. Dissertação (Mestrado em Engenharia Florestal) - Programa de Pós-graduação em Engenharia Florestal, Universidade Federal de Santa Maria, Santa Maria, RS.
OLIVEIRA, J.T.S.; SILVA, J.C. Variação radial da retratibilidade e densidade básica da madeira de Eucalyptus saligna Sm.. Revista Árvore, Viçosa, v.27, n.3, p.381-388, 2003.

SIAU, J.F. Wood: influence of moisture on physical properties. Virginia: Department of Wood Science and Forest Products, Virginia Polytechnic Institute and State University, 1995. 227p. Disponível em: <http://www.scielo.br/scielo.php?pid=S010067622003000300015\&script=sci_arttext $>$ Acesso 13 de agosto de 2012. doi: http://dx.doi.org/10.1590/S0100-67622003000300015.

SKAAR, C. Water in wood. Syracuse: Syracuse University, 1972. 218p.

STAMM, A.J. Wood and cellulose science. New York: The Ronald, 1964. 549p.

VOLVELLE, C.; MELLOTTEE, H. Modelisation de la pyrolyse oxydante ou noxydante de bois ou de déchets végetaux à partir de leurs composants. In: PALZ, W.; CHARTIER, P. (Eds.). Energy from biomass. 2.ed. London: Applied Sciences, 1982. p.925-931. 